\title{
Maintaining intraoperative normothermia reduces blood loss in patients undergoing major operations: a pilot randomized controlled clinical trial
}

\author{
Jie Yi, Hao Liang, Ruiyue Song, Hailu Xia and Yuguang Huang ${ }^{*}$ (B)
}

\begin{abstract}
Background: Inadvertent intraoperative hypothermia (core temperature $<36^{\circ} \mathrm{C}$ ) is a common but preventable adverse event. This study aimed to determine whether active intraoperative warming reduced bleeding in patients undergoing major operations: open thoracic surgery and hip replacement surgery.

Methods/Design: The study was a pilot, prospective, parallel two-arm randomized controlled trial. Eligible patients were randomly allocated to two groups: passive warming (PW), with application of a cotton blanket (thermal insulation), or active warming (AW), with a forced-air warming system. The primary endpoint was intraoperative blood loss, and secondary endpoints were surgical-site infection, cardiovascular events, and length of stay in the post-anesthesia care unit, intensive care unit, and hospital.

Results: Sixty-two patients were enrolled. Forced-air active warming maintained intraoperative normothermia in all AW subjects, whereas intraoperative hypothermia occurred in 21/32 (71.8\%) of PW patients $(p=0.000)$. The volume of blood loss was more in the PW group $(682 \pm 426 \mathrm{ml})$ than in the AW group $(464 \pm 324 \mathrm{ml})(p<0.021)$, and the perioperative hemoglobin value declined more in the PW group $(28.6 \pm 17.5 \mathrm{~g} / \mathrm{L})$ than in the AW group $(21.0 \pm 9.9 \mathrm{~g} / \mathrm{L})$ $(p=0.045)$. However, there were no difference in other clinical outcomes between two groups.
\end{abstract}

Conclusion: Intraoperative active warming is associated with less blood loss than passive warming in open thoracic and hip replacement operations in this pilot study.

Trial registration: This trial was registered with Clinicaltrials.gov (Identifier: NCT02214524) on 27 August 2014.

Keywords: Active forced warming, Passive warming, Intraoperative bleeding, Major surgery, Inadvertent intraoperative hypothermia

\section{Background}

Inadvertent perioperative hypothermia, defined as core temperature below $36{ }^{\circ} \mathrm{C}$ during operation, occurs commonly in patients undergoing general or regional anesthesia when their core body temperature is not regulated [1]. Core body temperature declines by as much as $1.6{ }^{\circ} \mathrm{C}$ within the first hour after the induction of anesthesia, which increases the risks of inadvertent hypothermia [2], mortality, blood

\footnotetext{
* Correspondence: yuguang_huang@hotmail.com

Department of Anesthesia, Peking Union Medical College Hospital, Chinese

Academy of Medical Science, 1 Shuaifuyuan, Dongcheng District, Beijing 100730, China
}

loss/blood transfusion [3-6], longer hospital stay [7], and surgical-site infection $[8,9]$.

Inadvertent hypothermia has been associated with intraoperative bleeding, but the relevant published data are inconclusive. Schmid et al. [4] evaluated blood loss and transfusion requirements in 60 patients undergoing primary total hip arthroplasties, who were randomly assigned to normothermia or mild hypothermia; intra- and post-operative blood loss were found significantly more in the hypothermic patients than in the normothermic patients, and a typical decrease in core temperature resulted in about $500 \mathrm{~mL}$ blood loss [4]. Winkler et al. [3] aggressively warmed patients to maintain a tympanic-membrane temperature of

(c) The Author(s). 2018 Open Access This article is distributed under the terms of the Creative Commons Attribution 4.0 International License (http://creativecommons.org/licenses/by/4.0/), which permits unrestricted use, distribution, and 
$36.5^{\circ} \mathrm{C}$ compared to core temperature of $36.0^{\circ} \mathrm{C}$ in patients receiving the conventional warming; they found that intraoperative blood loss was significantly more in the latter group. Besides increasing blood loss [10, 11], hypothermia can impair blood coagulation, as measured with activated partial thromboplastin time and bleeding time [12]. In a meta-analysis of 14 published randomized clinical trials comparing blood loss in normothermic and mildly hypothermic $\left(34-36^{\circ} \mathrm{C}\right)$ surgical patients, mild hypothermia $\left(<1{ }^{\circ} \mathrm{C}\right)$ was associated with significantly increased blood loss of about $16 \%(4-26 \%)$ and increased relative risk for transfusion of about 22\% (3-37\%) [5]. Although the above-cited authors have concluded that maintaining perioperative normothermia reduces blood loss and transfusion requirements [13] other data are not supportive [14, 15].

This study was part of a national perioperative health-care quality improvement and health economic research program in China. Limited data are available in China on whether AW reduces bleeding in patients undergoing major open operations. Thus, this pilot study was undertaken to evaluate objectively this issue in patients undergoing open thoracic or hip replacement surgery.

\section{Methods}

\section{Ethical status}

This study protocol was approved by the Institute Review Boards of Peking Union Medical College Hospital in Beijing, China.

\section{Study design}

This is a pilot prospective, parallel two-arm randomized controlled trial that is registered with clinicaltrials.gov (identifier: NCT02214524) in 2014. Due to the nature of intervention, it is an open-label study. Eligible subjects were those undergoing either initial unilateral total hip replacement or open thoracic operations (pulmonary lobectomy or esophagectomy). Inclusion criteria were: age18 to 80 years and American Society of Anesthesiologists Physical Status 1-3. Exclusion criteria were: history of excessive bleeding; partial thromboplastin time $>35 \mathrm{~s}$; prothrombin time $>35 \mathrm{~s}$; fibrinogen $<200 \mathrm{mg} / \mathrm{dL}$; platelet count $<100,000 / \mathrm{L}$; history of infection and fever within 4 weeks before surgery; use of steroid or immunosuppressant within 4 weeks before surgery; Raynaud's disease; hypothyroidism or hyperthyroidism; Uncontrolled insulin-dependent diabetes mellitus (preoperative glucose $>250 \mathrm{mg} / \mathrm{dL}$ ); preoperative temperature above $37.5^{\circ} \mathrm{C}$ or less than $36{ }^{\circ} \mathrm{C}$.

Patients eligible by the above criteria gave written, informed consent for participation in this study. Patients were enrolled and randomly allocated to either a passive warming (PW) group or an active warming (AW) group. Randomized numbers were generated by SAS 9.0 (SAS Institute, Cary, NC) and sealed in envelopes.

\section{PW group}

Patients were covered with unwarmed cotton blanket (thermal insulation) throughout the operation, from the preoperative holding area, operating room, to post-anesthesia care unit (PACU). Cover with unwarmed cotton blanket, usually one layer, is currently a routine practice in most institutions in China and can be considered standard of care.

\section{AW group}

Patients were covered with forced-air blankets connected to a warming unit (Bair-Hugger Patient Warmer, $3 \mathrm{M}$, St. Paul MN, USA), which has been reported to be the most effective warming device [16]. The blankets were placed on the operating room table and current turned on before the patient arrived. This protocol allowed the care provider to focus on the patient and be prepared from the start of the procedure. All patients were pre-warmed for 15-30 $\mathrm{min}$ with an upper-body blanket in the holding area and then moved onto the operating table prior to induction, where a pre-warmed lower-body blanket was already placed. The Bair-Hugger warming unit was set on its highest temperature $\left(43^{\circ} \mathrm{C}\right)$. If patient core temperature was below $37.5{ }^{\circ} \mathrm{C}$, the warming unit was placed on its highest temperature setting or switched to the appropriate setting at the discretion of investigators to maintain normothermia. This program was begun when patients arrived in the operating room waiting area and was terminated at the end of operation, when patients left the operating room and were transferred to the PACU or ICU.

\section{All patients received general anesthesia according to the following standard protocol}

The regimens were propofol $(2-2.5 \mathrm{mg} / \mathrm{kg})$, fentanyl $(2-$ $4 \mu \mathrm{g} / \mathrm{kg})$ and rocuronium $(0.8-1 \mathrm{mg} / \mathrm{kg})$ at induction, and sevoflurane (1.5-2 vol\%) mixed with $\mathrm{O}_{2} / \mathrm{N}_{2} \mathrm{O}(50 \% /$ $50 \%)$ for maintenance. The core temperature was measured with an infrared tympanic-membrane thermometer (ThermoScan PRO-4000, Braun GmbH, Kronberg, Germany). To avoid bias, the thermometer was calibrated and validated according to the manufacturer's manual before use. The core temperature was recorded every $15 \mathrm{~min}$ from the time the patient was placed in the operating room holding area until he/she was transported to the PACU after surgery. Intraoperative hypothermia was defined as core temperature below $36^{\circ} \mathrm{C}$ at any point in the perioperative period. All patients were followed for 30 days after surgery, especially for surgical-site infection. Investigators or study coordinators telephoned the patients or his/her family members if the patients had been already discharged from the hospital.

Primary and secondary endpoints, adverse events, and severe adverse events were collected for analysis. 


\section{Endpoints}

The primary endpoint was intraoperative blood loss. Intraoperative blood loss was estimated as the combined change in sponge weights (the density of a sponge is $1 \mathrm{~g} / \mathrm{ml}$ ) and volume of fluid in the suction reservoir, recorded by circulating nurses who were blinded to patients' core temperatures but not to whether the patients received AW. The need for blood transfusion was determined at physician discretion. The total volume of blood transfused included autologous transfusion and allogeneic transfusion, and the total volume was measured according to volume infused intravenously.

Secondary endpoints were postoperative surgical-site infection; cardiovascular events; length of stay (LOS) in the PACU, ICU and hospital; and shivering. The incidence and intensity of shivering were graded by the scale described by Crossley and Mahajan [17]: 0, no shivering; 1 , no visible muscle activity, but piloerection, peripheral vasoconstriction or both present (other causes excluded); 2 , muscular activity in only one muscle group; 3 , moderate muscular activity in more than one muscle group but no generalized shaking; and 4, violent muscular activity involving the whole body.

\section{Statistical analysis}

Sample-size estimation was based on reference to published articles $[4,5,15]$. As this is a pilot study, we planned to study a total of 60 subjects (30 per group) and then determine the sample size for a definitive study in the future.

Analyses were performed based on the intention-to-treat population, which included all patients except those who failed in the screening. Categorical data were analyzed with Chi-square or Fisher's exact test. Continuous variables were analyzed using unpaired, two-tailed t-tests or non-parametric analysis. $p$ value of less than 0.05 was considered to indicate a statistically significant difference. The data analysis was conducted with SAS 9.0 (SAS Institute, Cary, NC).

\section{Results}

\section{Baseline characteristics}

Sixty-four patients were screened with the inclusion and exclusion criteria after giving informed consent; 62 patients were eligible. Eligible patients were randomly allocated to a PW or AW group (Fig. 1). Table 1 depicts the baseline characteristics of patients enrolled. The two populations were similar in sex, age, body mass index, smoking history, past medical histories, type of operation, preoperative core temperature, and American Society of Anesthesiologists classification. Hemoglobin, platelet count, international normalized ratio, and fibrinogen values also were similar in the two groups.

\section{Intraoperative characteristics of the two patient groups}

Table 2 lists the intraoperative characteristics of the two patient groups, including data on the primary endpoint (intraoperative blood loss). The forced-air based active warming system maintained intraoperative normothermia in all AW patients ( $0 \%$ incidence of hypothermia) for the duration of operations. In contrast, the incidence of intraoperative hypothermia of $\leq 36.0{ }^{\circ} \mathrm{C}$ was $71.8 \%$ and of $\leq 35.5{ }^{\circ} \mathrm{C}$ was $37.5 \%$ in PW patients, and temperature reached $\leq 35{ }^{\circ} \mathrm{C}$ in three $(9.4 \%)$ PW patients.

The combined volume of intraoperative blood loss for the two operations (hip replacement and thoracic surgery) was significantly less in the AW group than in the PW group (464 $\pm 324 \mathrm{ml}$ vs $682 \pm 423 \mathrm{ml} ; p=0.0207)$.

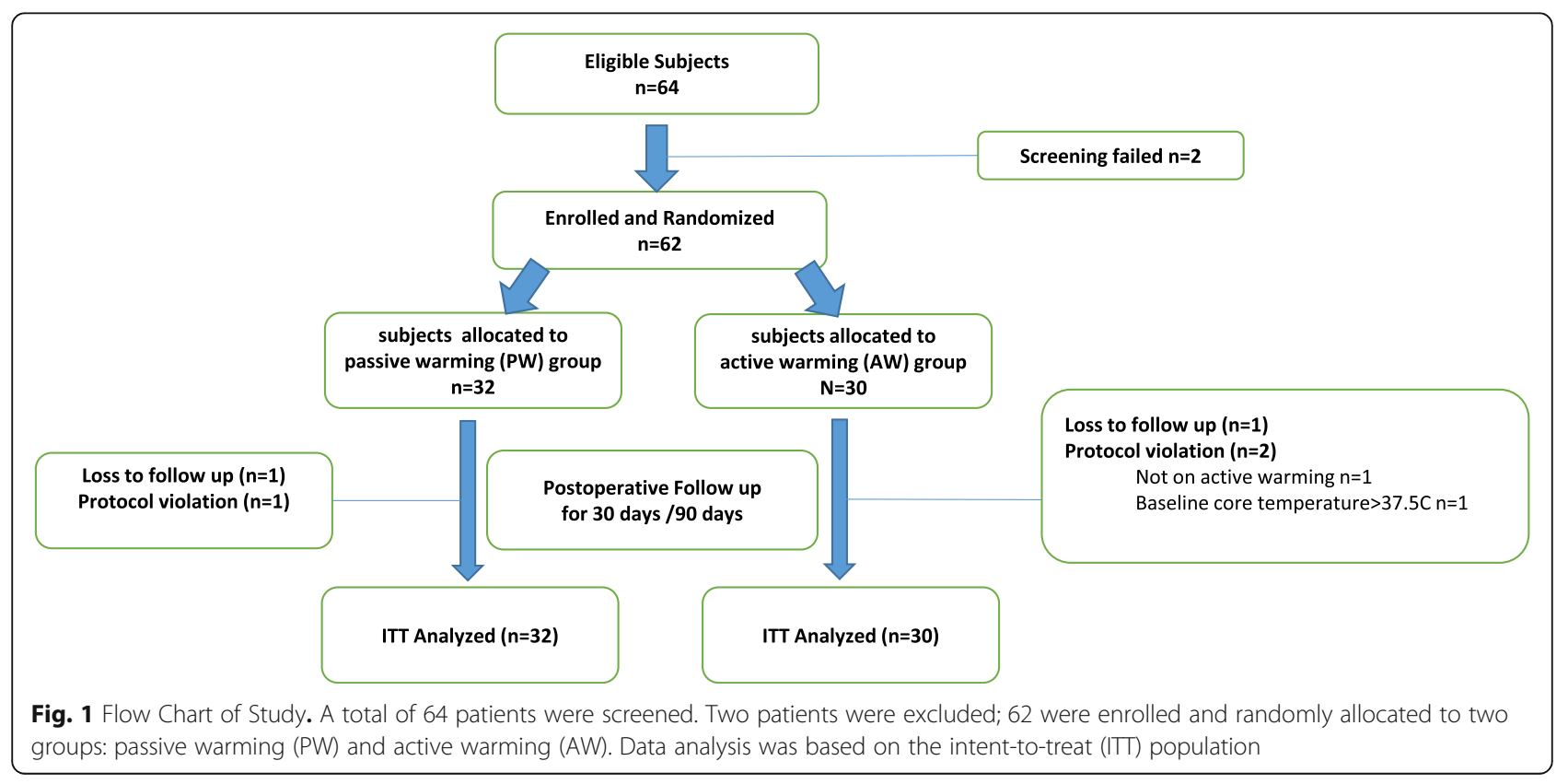


Table 1 Baseline Characteristics of Patients in Two Study

\begin{tabular}{|c|c|c|}
\hline Varables & $\begin{array}{l}\text { Passive } \\
\text { Warming } \\
\text { Group }(n=32)\end{array}$ & $\begin{array}{l}\text { Active } \\
\text { Warming } \\
\text { Group }(n=30)\end{array}$ \\
\hline Male, N (\%) & $25(78.1)$ & $21(70.0)$ \\
\hline Age, mean \pm std (year) & $58.5 \pm 11.5$ & $57.9 \pm 11.8$ \\
\hline BMI, mean \pm std & $24.7 \pm 3.8$ & $23.6 \pm 3.8$ \\
\hline History of smoking, N (\%) & $17(53.1)$ & $13(43.3)$ \\
\hline History of Alcholol, N (\%) & $16(50.0)$ & $17(56.7)$ \\
\hline \multicolumn{3}{|l|}{ Past medical history } \\
\hline Cardiovascular disease, N (\%) & $14(43.7)$ & $11(36.7)$ \\
\hline Celebrovascular disease, N (\%) & $3(9.3)$ & $0(0.0)$ \\
\hline Liver disease, $N(\%)$ & $3(9.3)$ & $3(10.0)$ \\
\hline Kidney disease, $N(\%)$ & $2(6.3)$ & $0(0.0)$ \\
\hline Diabetes, N (\%) & $5(15.7)$ & $4(13.3)$ \\
\hline \multicolumn{3}{|l|}{ Type of surgery } \\
\hline Hip replacement, $N(\%)$ & $12(38.7)$ & $12(40.0)$ \\
\hline Thoracic Surgery, N (\%) & $18(56.3)$ & $20(66.7)$ \\
\hline $\begin{array}{l}\text { Preoperative core temperature, mean } \pm \\
\operatorname{std}\left({ }^{\circ} \mathrm{C}\right)\end{array}$ & $37.2 \pm 0.3$ & $37.3 \pm 0.3$ \\
\hline \multicolumn{3}{|l|}{ ASA grade, $N(\%)$} \\
\hline Class 1 & $8(25.0)$ & $8(26.7)$ \\
\hline Class 2 & $22(68.7)$ & $21(70.0)$ \\
\hline Class 3 & $2(6.3)$ & $1(3.3)$ \\
\hline Hemoglobin, mean \pm std, (g/L) & $143.8 \pm 15.4$ & $137.4 \pm 16.5$ \\
\hline
\end{tabular}

Accordingly, hemoglobin values declined less from preoperative values to postoperative values in the AW group $(21.0 \pm 9.9 \mathrm{~g} / \mathrm{L})$ than in the PW group $(28.6 \pm$ $17.5 \mathrm{~g} / \mathrm{L})(p=0.0452)$. The proportion of patients that received blood transfusion in the PW group (11/32; $34.4 \%$ ) and the AW group (9/30; 30.0\%) were similar. The volume of blood transfused was similar between AW group $(137 \pm 294 \mathrm{ml})$ and PW group $(223 \pm 351 \mathrm{ml})$.

Ambient temperature, intravenous fluid administered, urine output, and volume of irrigation fluid used did not differ significantly between the two groups. Patients' platelet counts, prothrombin time, international normalized ratio, and fibrinogen values did not differ significantly from preoperative to postoperative determinations in either group (data not shown).

\section{Follow-up and adverse events}

Table 3 lists the postoperative outcomes (secondary endpoints) of the study. There was no difference between the two groups in LOS in the PACU, ICU or hospital. For evaluation of surgical-site infection, all patients were followed for 30 days after operation, and those who had an implant were followed for 90 days. Although three surgical-site infections were recorded in the PW group
(9.4\%) compared with none in the AW group, the difference is not statistically significant and is of unknown clinical significance. Shivering was recorded more often in PW patients than in AW patients, but the number of patients who had shivering was low, and difference was not statistically significant $(p=0.105)$. No cardiovascular events, including electrocardiographic abnormalities, were recorded in either patient group.

Only three patients experienced adverse events, and there was no statistically significant difference between the groups in the incidence of events (Table 4). One PW patient developed a postoperative pulmonary infection and another an anastomotic leak. One AW patient developed a pleural effusion. No severe adverse events were reported throughout the study.

\section{Discussion}

The adverse consequences of perioperative hypothermia have been well-established and investigated by the anesthesiology community worldwide. Besides anesthesiologists, nurses in charge of perioperative care, also have contributed to research in the area of perioperative hypothermia [13]. Maintaining normothermia is associated with enhanced recovery after surgery (ERAS) in patients, which is a multimodal, multidisciplinary approach (physicians, nurses, physician assistants, ICU workers etc.) to the care of the surgical patient [18]. Developed countries have many clinical practice guidelines and recommendations for the management of patients' body temperature during operations, but this is not the case in China, where lack of perioperative temperature management was first appreciated in the 1990s. Recently published data [19] have shown that the incidence of perioperative hypothermia was as high as $40 \%$ in Beijing, one of the most developed urban centers in China, with the most abundant healthcare resources.

The results of this study documented that AW was associated with reduced blood loss during two kinds of major surgery: open surgical thoracic and hip replacement operations. Although this association is not proven to be cause-effect, we cannot implicate with certainty other factors than warming, and the result is consistent with other reports ${ }^{4,5}$ of increased blood loss and transfusion requirement in patients who experienced hypothermia during major operations [20]. In a recent intraoperative hypothermia survey [19], patients who underwent thoracic surgery had a higher risk of intraoperative hypothermia and lower average temperature than did those undergoing other operations. Thus, in this study, we included patients receiving intrathoracic operations. We found that the Bair-Hugger forced-air (active) warming system maintained intraoperative normothermia, even in long operations, and reduced the intraoperative hypothermia rate to nil compared with about $72 \%$ 
Table 2 Intraoperative Characteristics of Patients in Two Study Groups

\begin{tabular}{|c|c|c|c|}
\hline Variable Name & Passive Warming Group $(n=32)$ & Active Warming Group $(n=30)$ & $p$ Value \\
\hline \multicolumn{4}{|l|}{ Core temperature (tympanic membranes) } \\
\hline$<36.0^{\circ} \mathrm{C}, \mathrm{N}(\%)<36.0^{\circ} \mathrm{C}, \mathrm{N}(\%)$ & $23(71.8)$ & $0(0.0)$ & 0.0000 \\
\hline$<35.5^{\circ} \mathrm{C}, \mathrm{N}(\%)<35.5^{\circ} \mathrm{C}, \mathrm{N}(\%)$ & $12(37.5)$ & $0(0.0)$ & 0.0001 \\
\hline$<35.0^{\circ} \mathrm{C}, \mathrm{N}(\%)<35.0^{\circ} \mathrm{C}, \mathrm{N}(\%)$ & $3(9.4)$ & $0(0.0)$ & 0.2384 \\
\hline \multicolumn{4}{|l|}{ Blood loss, mean \pm std (ml) } \\
\hline Total blood loss & $681.5 \pm 425.8$ & $464.0 \pm 324.1$ & \multirow[t]{3}{*}{0.0207} \\
\hline Hip repacement & $1035.4 \pm 491.4$ & $713.5 \pm 341.4$ & \\
\hline Thoracic surgery & $469.2 \pm 171.7$ & $311.5 \pm 199.0$ & \\
\hline \multicolumn{4}{|l|}{ Blood transfusion } \\
\hline Transfused patient, $N(\%)$ & $11(34.4)$ & $9(30.0)$ & 0.7124 \\
\hline Volume, mean \pm std $(\mathrm{ml})$ & $223.3 \pm 351.0$ & $137.1 \pm 293.9$ & 0.3008 \\
\hline Reduction of hemoglobin, mean \pm std $(\mathrm{g} / \mathrm{L})$ & $28.6 \pm 17.5$ & $21.0 \pm 9.9$ & 0.0452 \\
\hline \multicolumn{4}{|l|}{ Platelet } \\
\hline Preoperative, mean \pm std $\left(10^{9} / \mathrm{L}\right)$ & $210.6 \pm 59.9$ & $212.8 \pm 40.0$ & 0.8699 \\
\hline Postoperative, mean $\pm \operatorname{std}\left(10^{9} / \mathrm{L}\right)$ & $177.9 \pm 50.6$ & $177.8 \pm 40.4$ & 0.8839 \\
\hline \multicolumn{4}{|l|}{ Prothromin Time (PT) } \\
\hline Preoperative, mean \pm std (s) & $11.6 \pm 0.88$ & $11.3 \pm 0.58$ & 0.0439 \\
\hline Postoperative, mean \pm std (s) & $12.7 \pm 0.9$ & $12.6 \pm 0.6$ & 0.4158 \\
\hline \multicolumn{4}{|l|}{ International Normalized Ratio (INR) } \\
\hline Preoperative, mean \pm std & $1.0 \pm 0.1$ & $1.0 \pm 0.1$ & 0.0623 \\
\hline Postoperative, mean \pm std & $1.1 \pm 0.1$ & $1.0 \pm 0.1$ & 0.0815 \\
\hline \multicolumn{4}{|l|}{ Fibrinogen } \\
\hline Preoperative, mean \pm std $(\mathrm{g} / \mathrm{L})$ & $3.4 \pm 0.8$ & $3.2 \pm 0.9$ & 0.3367 \\
\hline Postoperative, mean \pm std $(\mathrm{g} / \mathrm{L}$ ) & $3.3 \pm 0.8$ & $3.8 \pm 1.4$ & 0.1247 \\
\hline Duration of surgery, mean \pm std (min) & $301.7 \pm 98.4$ & $248.3 \pm 118.8$ & 0.0579 \\
\hline Ambient temperature, mean $\pm \operatorname{std}\left({ }^{\circ} \mathrm{C}\right)$ & $21.1 \pm 1.2$ & $21.1 \pm 1.2$ & 0.9655 \\
\hline Intravenous fluid, mean \pm std (ml) & $2883.1 \pm 1037.1$ & $2652.3 \pm 1048.7$ & 0.3872 \\
\hline Urine output, mean \pm std (ml) & $948.4 \pm 827.8$ & $587.9 \pm 603.8$ & 0.0603 \\
\hline Irrigation fluid, mean \pm std (ml) & $3270.3 \pm 1187.4$ & $2898.2 \pm 1315.9$ & 0.2542 \\
\hline
\end{tabular}

Table 3 Postoperative Outcomes of Patients in Two Study Groups

\begin{tabular}{|c|c|c|c|}
\hline Variable & Passive Warming Group $(n=32)$ & Active Warming Group $(n=30)$ & $p$ Value \\
\hline \multicolumn{4}{|l|}{ PACU } \\
\hline Patient, N (\%) & $30(93.7)$ & $29(96.7)$ & 0.2056 \\
\hline LOS, mean \pm std (min) & $35.4 \pm 14.7$ & $28.7 \pm 21.8$ & 0.0525 \\
\hline \multicolumn{4}{|l|}{ ICU } \\
\hline Patient, N (\%) & $3(9.4)$ & $2(6.6)$ & 0.9468 \\
\hline LOS, mean \pm std (day) & $2.7 \pm 1.2$ & $7.0 \pm 2.8$ & 0.0545 \\
\hline Shivering, No of patients (\%) & $6(18.7)$ & $1(3.3)$ & 0.1048 \\
\hline Hospital LOS after surgery, mean \pm std (day) & $13.1 \pm 6.2$ & $11.1 \pm 4.9$ & 0.1989 \\
\hline Sugical Site Infetion, N (\%) & $3(9.4)^{\mathrm{a}}$ & $0(0)$ & 0.2384 \\
\hline Cardiovascular Events, N (\%) & $0(0.0)$ & $0(0.0)$ & \\
\hline
\end{tabular}


Table 4 Adverse Events and Severe Adverse Events ${ }^{a}$

\begin{tabular}{llll}
\hline Variable & $\begin{array}{l}\text { Passive Warming } \\
\text { Group }(n=29)\end{array}$ & $\begin{array}{l}\text { Active Warming } \\
\text { Group }(n=30)\end{array}$ & $p$ Value \\
\hline $\begin{array}{l}\text { Adverse Events, } \\
N(\%)\end{array}$ & $2(6.5)^{\mathrm{b}}$ & $1(3.3)^{c}$ & 0.5503 \\
$\begin{array}{l}\text { Severe Adverse } \\
\text { Events, } N(\%)\end{array}$ & $0(0.0)$ & $0(0.0)$ & \\
\hline
\end{tabular}

abased on safety data set

${ }^{b}$ One case is lung infection, another case anastomotic leak

'One case pleural effusion

in the PW group. Although the maintenance of intraoperative normothermia was associated with significantly less intraoperative blood loss in AW patients, a statistically significant increase in transfusion requirement was not found. This apparent discrepancy may be due to the limited sample size and the wide range in transfusion volumes in both AW and PW patients. Nonetheless, the possibility that maintaining normothermia reduces the perioperative requirements of allogeneic blood and blood products is important, as it could reduce the incidence of transfusion-related infections and allergic reactions as well cost, which are important considerations in China and other countries where the supplies of donated blood are limited.

Perioperative disturbances of hemostasis are common in patients undergoing major operations, and intraoperative hypothermia can induce platelet dysfunction [21]. In this study, though, we did not find reductions in either platelet counts or other measures of coagulation (prothrombin time, international normalized ratio or fibrinogen) in the PW group. The operative time was longer in the PW patients than in the AW patients by nearly $1 \mathrm{~h}$. Although this difference is not statistically significant, it raises the possibility that longer or more complicated operations was a determinant in our patients' blood loss. Thus, the cause of perioperative blood loss may have been more complicated than hypothermia in our patients.

We acknowledge that our study has limitations: 1 ) The sample size is small (62 cases). 2) The nature of the medical-device trial required that it be open-label; thus, the investigators and nurses and were not blinded to the method of warming, which could have introduced bias. To help lessen possible impact of this bias we measured patients' hemoglobin values as well as estimating blood loss through sponge weights and suction-reservoir volumes. 3) Due to small sample size, this study was underpowered to address the secondary endpoints of surgical-site infection, LOS and cardiovascular events.

In conclusion, we compared the intraoperative blood loss in patients who received major operations (open thoracic or hip replacement) and were randomized to passive warming, with a blanket, or active warming, with a forced-air warming system. Although this is a pilot study with small sample size, it was found that blood loss would be significantly more in patients treated with passive warming than in those treated with active warming. Since this was a pilot study as part of a national perioperative health-care quality improvement and health economic research program in China, a post-hoc health economic analysis and a pivotal trial-based health economic study may follow.

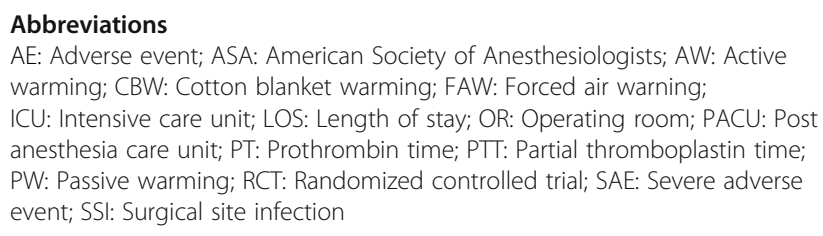
warming; CBW: Cotton blanket warming; FAW: Forced air warning; ICU: Intensive care unit; LOS: Length of stay; OR: Operating room; PACU: Post anesthesia care unit; PT: Prothrombin time; PTT: Partial thromboplastin time; PW: Passive warming; RCT: Randomized controlled trial; SAE: Severe adverse event; SSI: Surgical site infection

\section{Acknowledgements}

Authors thank Dr. William Brown, University of Colorado School of Medicine, Denver, CO, USA, for his scientifically reviewing and editing this manuscript.

\section{Funding}

This study was funded by the China National Health and Planned Parenthood Committee Health Quality Improvement Award (ID\# 201402017, principal investigator Yuguang Huang, MD), China National Nature Science Foundation (Grant\# 31070930, principal investigator Yuguang Huang, MD), and an unrestricted research grant (MDI0091, principal investigator Yuguang Huang, MD) from 3 M China.

\section{Availability of data and materials}

The anonymized dataset will be available by reasonable request sent to the corresponding author.

\section{Authors' contributions}

YH: funding, study conception \& design, administration, review and revision of data and manuscript; JY: study conception \& design, data collection, writing and drafting of manuscript; $\mathrm{HL}, \mathrm{RS}, \mathrm{HX}$ : patient recruitment, data collection and analysis; All authors read and approved the final manuscript.

\section{Ethics approval and consent to participate}

This study protocol was approved by Institute Review Boards (IRB) of Peking Union Medical College Hospital (PUMCH) in Beijing, China. Written informed consent was obtained from patients or surrogates prior to screening.

Consent for publication

Not applicable.

\section{Competing interests}

The authors declare that they have no competing interests.

\section{Publisher's Note}

Springer Nature remains neutral with regard to jurisdictional claims in published maps and institutional affiliations.

Received: 12 December 2017 Accepted: 21 August 2018

Published online: 08 September 2018

\section{References}

1. Sessler DI. Mild perioperative hypothermia. N Engl J Med. 1997; 336(24):1730-7.

2. Lenhardt R. Monitoring and thermal management. Best Pract Res Clin Anaesthesiol. 2003;17(4):569-81.

3. Winkler M, Akca O, Birkenberg B, Hetz H, Scheck T, Arkilic CF, Kabon B, Marker E, Grubl A, Czepan R, et al. Aggressive warming reduces blood loss during hip arthroplasty. Anesth Analg. 2000;91(4):978-84.

4. Schmied H, Kurz A, Sessler DI, Kozek S, Reiter A. Mild hypothermia increases blood loss and transfusion requirements during total hip arthroplasty. Lancet. 1996;347(8997):289-92. 
5. Rajagopalan S, Mascha E, Na J, Sessler DI. The effects of mild perioperative hypothermia on blood loss and transfusion requirement. Anesthesiology. 2008;108(1):71-7.

6. Leijtens B, Koeter M, Kremers K, Koeter S. High incidence of postoperative hypothermia in total knee and total hip arthroplasty: a prospective observational study. J Arthroplast. 2013;28(6):895-8.

7. Kurz A, Sessler DI, Lenhardt R. Perioperative normothermia to reduce the incidence of surgical-wound infection and shorten hospitalization. Study of wound infection and temperature group. N Engl J Med. 1996;334(19):1209-15.

8. Aasen AO, Barie PS, Faist E, Ford HR, Fry DE, Hau T. Panel discussion: current issues in the prevention and management of surgical site infection--part 1. Surg Infect. 2002;3(Suppl 1):S1-7.

9. Aasen AO, Barie PS, Faist E, Ford HR, Fry DE, Hau T. Panel discussion: current issues in the prevention and management of surgical site infection--part 2. Surg Infect. 2002;3(Suppl 1):S99-102.

10. Pu Y, Cen G, Sun J, Gong J, Zhang Y, Zhang M, Wu X, Zhang J, Qiu Z, Fang F. Warming with an underbody warming system reduces intraoperative hypothermia in patients undergoing laparoscopic gastrointestinal surgery: a randomized controlled study. Int J Nurs Stud. 2014;51(2):181-9.

11. Long KC, Tanner EJ, Frey M, Leitao MM Jr, Levine DA, Gardner GJ, Sonoda Y, Abu-Rustum NR, Barakat RR, Chi DS. Intraoperative hypothermia during primary surgical cytoreduction for advanced ovarian cancer: risk factors and associations with postoperative morbidity. Gynecol Oncol. 2013;131(3):525-30.

12. Cavallini M, Baruffaldi Preis FW, Casati A. Effects of mild hypothermia on blood coagulation in patients undergoing elective plastic surgery. Plast Reconstr Surg. 2005;116(1):316-21. discussion 322-313

13. Tappen RM, Andre SP. Inadvertent hypothermia in elderly surgical patients. AORN J. 1996;63(3):639-44.

14. Schur MD, Blumstein GW, Seehausen DA, Ross PA, Andras LM, Skaggs DL. Intraoperative hypothermia is common, but not associated with blood loss or transfusion in pediatric posterior spinal fusion. J Pediatr Orthop. 2016;

15. Johansson T, Lisander B, Ivarsson I. Mild hypothermia does not increase blood loss during total hip arthroplasty. Acta Anaesthesiol Scand. 1999; 43(10):1005-10.

16. Hynson JM, Sessler DI. Intraoperative warming therapies: a comparison of three devices. J Clin Anesth. 1992;4(3):194-9.

17. Crossley AW, Mahajan RP. The intensity of postoperative shivering is unrelated to axillary temperature. Anaesthesia. 1994;49(3):205-7.

18. Ljungqvist O, Scott M, Fearon KC. Enhanced recovery after surgery: a review. JAMA Surg. 2017;152(3):292-8.

19. Yi J, Xiang Z, Deng X, Fan T, Fu R, Geng W, Guo R, He N, Li C, Li L, et al. Incidence of inadvertent intraoperative hypothermia and its risk factors in patients undergoing general anesthesia in Beijing: a prospective regional survey. PLoS One. 2015;10(9):e0136136.

20. Burger L, Fitzpatrick J. Prevention of inadvertent perioperative hypothermia. Br J Nurs. 2009:18(18):1114, 1116-9.

21. Valeri CR, Khabbaz K, Khuri SF, Marquardt C, Ragno G, Feingold H, Gray AD, Axford T. Effect of skin temperature on platelet function in patients undergoing extracorporeal bypass. J Thorac Cardiovasc Surg. 1992;104(1):108-16.

Ready to submit your research? Choose BMC and benefit from:

- fast, convenient online submission

- thorough peer review by experienced researchers in your field

- rapid publication on acceptance

- support for research data, including large and complex data types

- gold Open Access which fosters wider collaboration and increased citations

- maximum visibility for your research: over $100 \mathrm{M}$ website views per year

At BMC, research is always in progress.

Learn more biomedcentral.com/submissions 